\title{
Frequent concomitant presence of Desulfitobacterium spp. and "Dehalococcoides" spp. in chloroethene-dechlorinating microbial communities
}

\author{
Katia Rouzeau-Szynalski • Julien Maillard • \\ Christof Holliger
}

Received: 24 October 2010 /Revised: 23 November 2010 /Accepted: 24 November 2010 /Published online: 9 December 2010

(C) Springer-Verlag 2010

\begin{abstract}
The presence of chloroethene dechlorination activity as well as several bacterial genera containing mainly organohalide-respiring members was investigated in 34 environmental samples from 18 different sites. Cultures inoculated with these environmental samples on tetrachloroethene and amended weekly with a seven organic electron donor mixture resulted in 11 enrichments with cis-DCE, ten with $\mathrm{VC}$, and 11 with ethene as dechlorination end product, and only two where no dechlorination was observed. "Dehalococcoides" spp. and Desulfitobacterium spp. were detected in the majority of the environmental samples independently of the dechlorination end product formed. The concomitant presence of Dehalococcoides spp. and Desulfitobacterium spp. in the majority of the enrichments suggested that chloroethene dechlorination was probably the result of catalysis by at least two organohalide-respiring genera either in parallel or by stepwise catalysis. A more detailed study of one enrichment on cis-DCE suggested that in this culture Desulfitobacterium spp. as well as Dehalococcoides spp. dechlorinated cis-DCE whereas dechlorination of $\mathrm{VC}$ was only catalyzed by the latter.
\end{abstract}

Electronic supplementary material The online version of this article (doi:10.1007/s00253-010-3042-0) contains supplementary material, which is available to authorized users.

K. Rouzeau-Szynalski $\cdot$ J. Maillard $\cdot$ C. Holliger $(\bowtie)$ Ecole Polytechnique Fédérale de Lausanne (EPFL),

ENAC Laboratory for Environmental Biotechnology,

Station 6,

CH-1015, Lausanne, Switzerland

e-mail: christof.holliger@epfl.ch

Present Address:

K. Rouzeau-Szynalski

Product Technology Center-Nestlé,

Konolfingen, Switzerland
Keywords Organohalide respiration · Dehalorespiration . Halorespiration · Reductive dechlorination · Bioremediation

\section{Introduction}

Since decades chloroethenes, such as tetrachloroethene (PCE) and trichloroethene (TCE), are produced in large quantities worldwide and as a result of inappropriate handling or storage, they are very frequently encountered as contaminants in groundwater, soils, and sediments (McCarty 1997; Fetzner 1998; Comber and Gardner 1999). Under anaerobic conditions, these compounds can be reductively dechlorinated via dichloroethene (DCE) and vinyl chloride (VC) to harmless ethene by organohaliderespiring bacteria for which chloroethenes act as electron acceptors in the energy metabolism (Holliger et al. 1999). While cis-1,2-DCE has been mainly reported as dechlorination intermediate, some studies have also shown that trans-DCE is sometimes formed (Gerritse et al. 1999; Griffin et al. 2004; Miller et al. 2005; Cheng et al. 2010).

The question was, after isolation of several organohaliderespiring bacteria with different metabolic properties, whether these bacteria are also relevant in the environment where natural attenuation of chloroethenes occurs. A survey including samples from the United States of America as well as Europe has shown that "Dehalococcoides" spp. seemed to be widespread and often detected where ethene formation has been observed (Hendrickson et al. 2002). Another study has shown that the genus Desulfitobacterium could be detected at many different locations in Quebec, Canada (Lanthier et al. 2001). Other organohalide-respiring bacteria such as $G$. lovleyi and Dehalobacter spp. have also been observed in groundwater systems and enrichments cultures thereof (Davis et al. 2002; Grostern and Edwards 2006; Amos et 
al. 2007; Daprato et al. 2007; Nijenhuis et al. 2007; Dowideit et al. 2010).

The goal of this study was to evaluate the presence of bacterial genera such as Desulfitobacterium, Dehalobacter, and Dehalococcoides among which most isolates are organohalide-respiring bacteria in numerous environmental samples and enrichment cultures thereof and to link the presence or absence of these genera with the dechlorination end product formed.

\section{Material and methods}

Chemicals All chemicals were analytical grade and used without purification. PCE (99\%) and n-hexadecane (99\%) were purchased from Acros Organics, Geel, Belgium. Cis-1,2dichloroethene (cis-DCE) (97\%) was obtained from SigmaAldrich, Chemie GmbH, Steinheim, Germany. VC (99.5\%) and ethene (99.95\%) were procured from Fluka AG, Buchs, Switzerland. All gasses $\left(\mathrm{N}_{2}, \mathrm{CO}_{2}, \mathrm{H}_{2}\right)$ were purchased from SLGas, Sauerstoffwerk Lenzburg, Switzerland. Due to the possible presence of inhibiting compounds in synthetic cisDCE, also biogenic cis-DCE was used prepared as described by Maymo-Gatell et al. (2001), however Desulfitobacterium hafniense strain TCE1 was used here as PCE-dechlorinating bacterium.

Source of inocula and enrichment cultures The 34 inocula used in this study were from 18 different sites and comprised the following: 19 wet aquifer samples, eight groundwater samples, two sludge samples from bioreactors treating chlorinated compound-contaminated waters, and five non-contaminated samples (one mix of different digester sludge from municipal wastewater treatment plants, two river and two lake sediment samples) (see Online Resource 1-Table S1).

The enrichment cultures in 100 or $500 \mathrm{ml}$ serum bottles (VWR international AG, Merck, Dietikon, Switzerland) containing 50 or $250 \mathrm{ml}$ of anaerobic medium were performed with a two-liquid phase system that has been described previously (Holliger et al. 1998). In an anaerobic glove box (Anaerobic System model 1,024, Forma Scientific, Brouwer, Luzern, Switzerland), the 100$\mathrm{ml}$ bottles containing freshly prepared anaerobic mineral medium were inoculated by the addition of approximately $1 \mathrm{~g}$ of solid material or $10 \mathrm{ml}$ liquid (water or sludge), depending on the inoculum. Oxygen was removed from the anaerobic glove box with R-20 palladium catalyst provided by BASF, Wädenswil, Switzerland. The bottles were closed in the glove box and the gas phase of the bottles was aseptically replaced with a $\mathrm{N}_{2} / \mathrm{CO}_{2}$ mixture (4:1, v/v, 1.0 bar over pressure) using a gas exchange system (Druva Sonderventie GmbH, Eppelheim, German) and sterile hydrophobic $0.2 \mu \mathrm{m}$ filters (Sarstedt AG, Sevelen, Switzerland). PCE, TCE, and DCE were dissolved in hexadecane at a concentration of 100,80 , and $50 \mathrm{mM}$, respectively. The hexadecane solutions were added to serum bottles that were sealed with Viton rubber stoppers. After changing the gas phase with pure nitrogen, the hexadecane solutions were sterilized by heat $\left(121^{\circ} \mathrm{C}\right.$, $20 \mathrm{~min}$ ). The volumes added to inoculated culture bottles by syringe were (per $50 \mathrm{ml}$ of medium); PCE $5 \mathrm{ml}$, TCE $6 \mathrm{ml}$, DCE $10 \mathrm{ml}$. Vinyl chloride was injected as pure gas directly to the culture bottles in amounts of approximately $1 \mathrm{mmol}(25 \mathrm{ml})$ per $50 \mathrm{ml}$ of medium. These latter cultures did not contain any hexadecane.

Electron donors were added once a week from 50 times concentrated anoxic stock solutions. A mixture of seven electron donors (acetate-formate-propionate-butyrate-ethanollactate-pyruvate) was mainly used, each added at a concentration of $0.285 \mathrm{mM}$ resulting in a total concentration of $2 \mathrm{mM}$ total electron donor per week. For the cis-DCE dechlorinating AQ1 culture, an electron donor mixture containing propionate, butyrate and ethanol $(0.67 \mathrm{mM}$ each) was used. The enrichment cultures were incubated stationary at $30^{\circ} \mathrm{C}$ in the dark.

Analytical methods The dechlorination activity of the enrichments was followed by measuring the concentration of chloride in the aqueous phase by silver ion titration with a Chlor-o-counter (Flohr Instrument, Nieuwegein, Netherlands). Chloroethenes and methane were analyzed by gas chromatography as previously described (Maillard et al. 2003). The volatile fatty acids were analyzed using a HPLC (Varian Star 9,100, Varian AG, Zug, Switzerland) with a refractive index detector (ERC7,415 A, Varian). Samples of $1.2 \mathrm{ml}$ were deproteinated by adding $150 \mu \mathrm{l} \mathrm{Ba}(\mathrm{OH})_{2} \quad 0.15 \mathrm{M}$ and $150 \mu \mathrm{l} \mathrm{ZnSO}_{4}$ $0.15 \mathrm{M}$, centrifuging $10 \mathrm{~min}$ at $11,000 \times \mathrm{g}$, and filtering with a $0.2-\mu \mathrm{m}$ filter (Sarsted, Sevelen, Switzerland). The HPLC was equipped with a guard column connected to an organic acid column maintained at $60{ }^{\circ} \mathrm{C}$ (ORH-801, InterAction INC, Varian). The solvent was $50 \mathrm{mM} \mathrm{H}_{2} \mathrm{SO}_{4}$ with a flow rate of $0.61 \mathrm{ml} / \mathrm{min}$.

DNA extraction The Kit UltraClean ${ }^{\mathrm{TM}}$ Soil DNA Isolation from MoBio Laboratories, Inc. (BIOzym, Landgraaf, Netherlands) was used for the DNA extraction. Aliquots of $0.5 \mathrm{~g}$ of soil and aquifer and $10 \mathrm{ml}$ of environmental water sample, and $15 \mathrm{ml}$ of the enrichment cultures were extracted. Liquid samples were centrifuged at $29,000 \times \mathrm{g}$ for $5 \mathrm{~min}$ at $4{ }^{\circ} \mathrm{C}$, the pellet resuspended in $300 \mu \mathrm{l} 0.1 \mathrm{M}$ sodium phosphate buffer (pH 8.0), and the suspension transferred in the extraction tubes provided by MoBio. Bead beating was performed two times $30 \mathrm{~s}$ at $1,000 \mathrm{rpm}$ with a Mikrodismembrator $\mathrm{S}$ 
(B. Braun Biotech International $\mathrm{GmbH}$, Melsungen, Germany). The DNA was extracted according the supplier's protocol and recovered finally in $50-\mu l$ samples.

End-point PCR detection and quantitative real-time PCR Specific primers (Online Resource 1-Table S2) used for the detection of known PCE-dechlorinating bacterial species and genera were designed together with people from Bioclear BV (Groningen, Netherlands) in the framework of the EU FP5 project MAROC. Primers for the detection of $b v c A$ and $v c r A$ genes were taken from (Behrens et al. 2008). All primers were purchased at Microsynth AG (Balgach, Switzerland). Amplification reactions were performed with a thermocycler T3 (Biometra, Biolabo, Châtel-St-Denis, Switzerland). The $10 \mu \mathrm{l}$ PCR mixture contained $1 \times$ PCR Buffer B, $0.2 \mathrm{mM}$ dNTPs, $0.5 \mu \mathrm{M}$ of each primer, $0.1 \mu \mathrm{l}(5 \mathrm{U} / \mu \mathrm{l})$ of Taq polymerase (Promega, Catalys AG, Wallisellen, Switzerland), and $1 \mu \mathrm{l}$ of the extracted DNA. The following thermocycling program was used for all primer sets except for Desulfitobacterium spp.; $94{ }^{\circ} \mathrm{C}$ for $3 \mathrm{~min}$ (one cycle); $94{ }^{\circ} \mathrm{C}$ for $30 \mathrm{~s}$, annealing temperature (Table S2) for $30 \mathrm{~s}, 72^{\circ} \mathrm{C}$ for $1 \mathrm{~min} 30 \mathrm{~s}$ ( 25 of 35 cycles); $72^{\circ} \mathrm{C}$ for $10 \mathrm{~min}$ (one cycle). For Desulfitobacterium spp., the elongation was only $30 \mathrm{~s}$ instead of $1 \mathrm{~min} 30 \mathrm{~s}$. For DNA isolated from the inocula, the PCR comprised 35 cycles whereas only 25 cycles were used for enrichment DNA.

Quantitative real-time PCR was carried out as described previously with the primers specifically developed for this purpose (Smits et al. 2004). Here, the $16 \mathrm{~S}$ rRNA gene of Desulfitobacterium spp. and Dehalococcoides spp. were targeted.

T-RFLP analysis The $16 \mathrm{~S}$ rRNA genes of the microbial communities present in the samples were amplified with general bacterial primers Eub 8 F-Hex ${ }^{\mathrm{TM}}$ fluorescently labeled at the $5^{\prime}$ position and Eub 534R (Online Resource 1 - Table S2). Two tubes of $50 \mu 1$ PCR mixture for each sample contained 1× PCR buffer B (Promega), $0.2 \mathrm{mM}$ dNTPs, $0.24 \mu \mathrm{M}$ of each primers (forward and reverse primers), $0.4 \mu \mathrm{l}(5 \mathrm{U} / \mu \mathrm{l})$ of Taq polymerase, and $2 \mathrm{ng}$ of the extracted DNA. The following thermocycling program was used; $94{ }^{\circ} \mathrm{C}$ for $5 \mathrm{~min}$ (one cycle); $94{ }^{\circ} \mathrm{C}$ for $30 \mathrm{~s}, 56{ }^{\circ} \mathrm{C}$ for $45 \mathrm{~s}, 72{ }^{\circ} \mathrm{C}$ for $2 \mathrm{~min}(35 \mathrm{cycles}) ; 72{ }^{\circ} \mathrm{C}$ for $10 \mathrm{~min}$ (one cycle). The two tubes of each sample were pooled, purified with the QIAquick PCR purification Kit (Qiagen AG, Basel, Switzerland) and resuspended in $30 \mu l$. The purified PCR products were quantified with a fluorometer (TD 700 Tuner Designs, Witec AG, Littau, Switzerland) using the PicoGreen kit (PicoGreen ${ }^{\circledR}$ dsDNA quantification Kit, Molecular Probes Europe B.V., Leiden, Netherlands) and $50 \mathrm{ng}$ were digested with the enzyme HaeIII (Promega) during $4 \mathrm{~h}$ at $37{ }^{\circ} \mathrm{C}$. An aliquot of $1 \mu \mathrm{l}$ of the digested product was prepared according to the supplier $\mathrm{ABI}$ (Applied Biosystems, Rotkreuz, Switzerland) and loaded in the $\mathrm{ABI}$ Prism ${ }^{\circledR} 3,100$ capillary array $(50 \mathrm{~cm})$ and ABI Prism ${ }^{\circledR} 3,100$ POP- $6^{\text {TM }}$ polymer. The results were analyzed with the package ABI Prism ${ }^{\circledR}$ 3,100 GeneScan Software. Each peak of the electropherogram represented a molecular operational taxonomic unit (OTU) which can originate from one strain, a species, different members of a genus, or any other unit of classification. The results were analyzed with the online software TAP T-RFLP from Ribosomal Database Project II (Maidak et al. 1999). To estimate the relative abundance of an OTU, its peak area was expressed as the percentile of the total of all peak areas of the electropherogram.

Cloning and sequencing The detail procedure is described in the supplementary material (Online Resource 1). Briefly, PCR products targeting bacterial $16 \mathrm{~S}$ rRNA genes were purified, ligated into pGEM-T Easy vector (Promega), and transformed into competent Escherichia coli $\mathrm{DH} 5 \alpha$ cells by heat shock. Colony PCR was done with T7 and SP6 promoter primers. Clones containing DNA fragments of expected length were sequenced with T7 and SP6 promoter primers using the BigDye Terminator v3.0 Ready Reaction Kit (ABI Applied Biosystems) according to the manufacturer's instructions. Samples were run on the ABI Prism 3,100 Genetic Analyzer.

Sequences were analyzed with the software package Lasergene (DNASTAR, Inc, Madison, WI, USA) and aligned with ClustalX (Thompson et al. 1994). The phylogenetic trees were designed using the software package MEGA4 (Tamura et al. 2007). The sequences were compared with databases using the BLAST software (Altschul et al. 1990).

Nucleotide sequences The obtained sequences for the AQ1 clones were submitted to Genbank with the following reference numbers: HM63539-HM635368.

\section{Results}

Presence of known organohalide-respiring bacteria in numerous inocula and cultures thereof In the framework of different projects, a total of 34 environmental samples were collected from 18 different sites. The majority of the samples originated from sites contaminated with chlorinated compounds (Online Resource 1-Table S1). The environmental samples were used as inocula for enrichment cultures amended with PCE as electron acceptor and a substrate mixture composed of seven organic compounds (acetate-formate-propionate-butyrate- 
ethanol-lactate-pyruvate) that was added once a week. Chloride production started in all cultures quite rapidly without considerable lag phases (Fig. 1). PCE dechlorination to cis-DCE was typically achieved after 7-9 weeks of incubation and no formation of trans-DCE was observed. Chloride continued to be formed in cultures producing VC and ethene but at lower rates (Fig. 1). However, formation of $\mathrm{VC}$ and ethene was not observed in all enrichments. In 11 cultures, cis-DCE was the dechlorination end product, $\mathrm{VC}$ in another ten, and in 11 it was ethene (Table 1). Only in two cultures no dechlorination was observed.

End-point PCR used for the detection of genera Dehalococcoides, Desulfitobacterium, and Dehalobacter showed the presence of Dehalococcoides in 23 inocula, Desulfitobacterium in 21, and Dehalobacter only in seven (Table 1). Dehalococcoides was also detected in most enrichments, even in those where dechlorination did not go beyond cis-DCE, but was below the detection limit in three enrichments where ethene was formed (Table 1). Desulfitobacterium was detected in all enrichments but one where dechlorination occurred whereas Dehalobacter was only detected in ten (Table 1).

Analysis of an enrichment culture dechlorinating DCE to ethene One of the cultures obtained from the inoculum AQ1 with cis-DCE as electron acceptor instead of PCE was analyzed in more detail. It was fed with a propionatebutyrate-ethanol electron donor mixture and transferred approximately once in every 6-7 weeks.

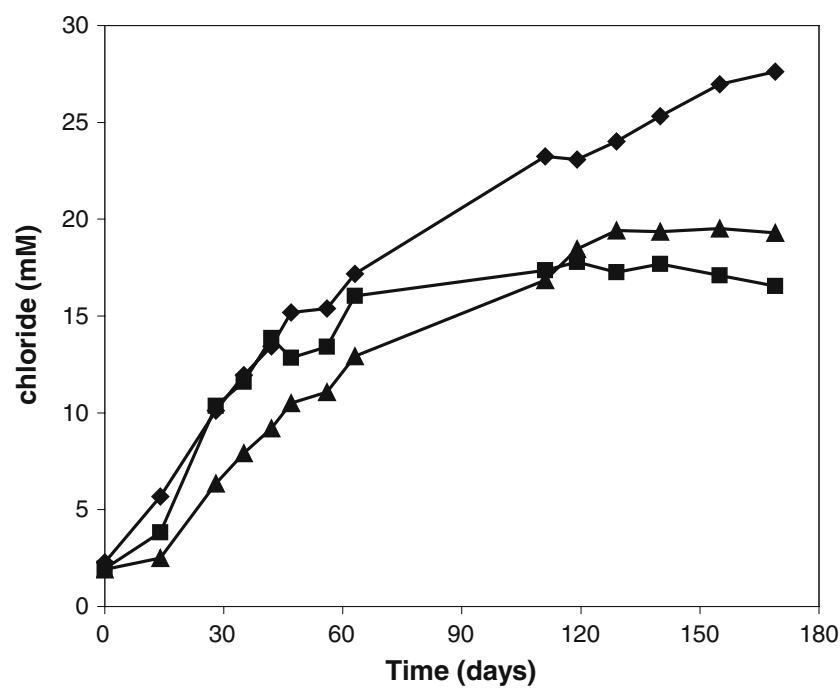

Fig. 1 Chloride production in cultures inoculated with different environmental samples amended with a seven electron donor mixture once a week and with hexadecane containing $100 \mathrm{mM}$ PCE (nominal PCE concentration in water phase ca. $8 \mathrm{mM}$ ), and producing different dechlorination end products. Culture from sample GW3 (filled square) producing DCE, from sample GW5 (filled triangle) producing VC, and from sample AQ5 (filled diamond) producing ethene
After more than ten transfers and up-scaling from 50 to $250 \mathrm{ml}$ cultures, cultures were inoculated containing either PCE or $c i s$-DCE as chlorinated electron acceptor. Cis-DCE was dechlorinated in a two-step process with an initial accumulation of $\mathrm{VC}$ and no formation of ethene until almost all cis-DCE was consumed followed by the dechlorination of $\mathrm{VC}$ to ethene (Fig. 2). Ethanol was completely consumed between two weekly electron donor additions whereas propionate and butyrate were only partially consumed. Hydrogen and acetate balances indicated that all hydrogen formed upon syntrophic electron donor oxidation was consumed by reductive dechlorination and methanogenesis and that no or almost no homoacetogenesis took place in this enrichment culture (Online Resource 1-Fig. S1). PCE was also dechlorinated by this culture line but slowly, and the formed cis-DCE was further dechlorinated to $\mathrm{VC}$ and some ethene although large amounts of PCE were still present (Online Resource 1Fig. S2).

Cloning and sequencing of bacterial $16 \mathrm{~S}$ rRNA genes amplified from DNA isolated from the initial AQ1 culture on the propionate-butyrate-ethanol mixture and cis-DCE, showed the presence of Desulfitobacterium spp. and Dehalococcoides spp. in this microbial consortium. Twelve of the 30 clones classified with the bacterial genus Dehalococcoides and five with Desulfitobacterium spp. (Online Resource 1-Fig. S3). The closest relatives of the Dehalococcoides-affiliated clones were the strains GT, CBDB1, FL2, and BAV1 with 99\% sequence identity. The closest relative to the Desulfitobacterium-affiliated clones was the first $16 \mathrm{~S}$ rRNA gene copy of strain DCB2 (Dhaf_R0006, 98\% identity), with all of these five clones corresponding to the shortest version of Desulfitobacterium 16 S rRNA genes (Online Resource 1-Fig. S3). The other clones affiliated with Wolinella spp. (seven clones, 100\%), Azovibrio spp. (two clones, 100\%), or Bacteroidetes (one clone, $100 \%)$. Three additional clones were only distantly related to Treponema spp. (75\%).

The analysis of samples taken during 6 weeks of incubation suggested that cis-DCE was dechlorinated by Desulfitobacterium spp. and Dehalococcoides spp. whereas VC was dechlorinated by Dehalococcoides spp. (Fig. 2). On successive transfers, Desulfitobacterium spp. was systematically and abundantly present during the first 2 weeks of incubation whereas Dehalococcoides dominated the consortium after 4-6 weeks of incubation.

\section{Discussion}

The concomitant presence of Dehalococcoides spp. and Desulfitobacterium spp. in the majority of the samples 
Table 1 End-point PCR detection of Dehalococcoides, Desulfitobacterium, and Dehalobacter in inocula and enrichments thereof amended with a seven electron donor mixture added weekly and containing PCE dissolved in hexadecane

\begin{tabular}{|c|c|c|c|c|c|c|c|c|}
\hline \multirow[t]{2}{*}{ End product ${ }^{\mathrm{a}}$} & \multirow[t]{2}{*}{ Site $^{\mathrm{b}}$} & \multirow{2}{*}{ Sample $^{\mathrm{b}}$} & \multicolumn{2}{|c|}{ Dehalococcoides } & \multicolumn{2}{|c|}{ Desulfitobacterium } & \multicolumn{2}{|c|}{ Dehalobacter } \\
\hline & & & Inoculum & Enrichment & Inoculum & Enrichment & Inoculum & Enrichment \\
\hline \multirow[t]{12}{*}{ DCE } & S3 & GW1 & $\mathrm{X}$ & $\mathrm{X}$ & $\mathrm{X}$ & $\mathrm{X}$ & $\mathrm{X}$ & \\
\hline & S4 & AQ8 & $\mathrm{X}$ & $\mathrm{X}$ & $\mathrm{X}$ & $\mathrm{X}$ & & $\mathrm{X}$ \\
\hline & S4 & GW2 & $\mathrm{X}$ & $\mathrm{X}$ & $\mathrm{X}$ & $\mathrm{X}$ & & \\
\hline & S5 & AQ9 & $\mathrm{X}$ & $\mathrm{X}$ & $\mathrm{X}$ & $\mathrm{X}$ & & \\
\hline & S5 & GW3 & $\mathrm{X}$ & & $\mathrm{X}$ & $\mathrm{X}$ & & \\
\hline & S7 & GW6 & $\mathrm{X}$ & $\mathrm{X}$ & $\mathrm{X}$ & $\mathrm{X}$ & $\mathrm{X}$ & \\
\hline & S7 & GW7 & $X$ & $\mathrm{X}$ & $\mathrm{X}$ & $\mathrm{X}$ & & \\
\hline & S13 & AQ14 & $\mathrm{X}$ & & $\mathrm{X}$ & $\mathrm{X}$ & $\mathrm{X}$ & $\mathrm{X}$ \\
\hline & S14 & AQ16 & & $\mathrm{X}$ & & $\mathrm{X}$ & & \\
\hline & S14 & AQ17 & & $\mathrm{X}$ & $\mathrm{X}$ & $\mathrm{X}$ & & \\
\hline & S14 & AQ18 & & & & $\mathrm{X}$ & & \\
\hline & No. of samples & 11 & 8 & 8 & 9 & 11 & 3 & 2 \\
\hline \multirow[t]{11}{*}{$\mathrm{VC}$} & S1 & AQ3 & $\mathrm{X}$ & $\mathrm{X}$ & & $\mathrm{X}$ & & \\
\hline & S3 & AQ6 & $\mathrm{X}$ & & & $\mathrm{X}$ & & \\
\hline & S4 & AQ4 & $\mathrm{X}$ & $\mathrm{X}$ & $\mathrm{X}$ & $\mathrm{X}$ & & \\
\hline & S5 & GW4 & $\mathrm{X}$ & $\mathrm{X}$ & $\mathrm{X}$ & $\mathrm{X}$ & & \\
\hline & S6 & GW5 & $\mathrm{X}$ & $\mathrm{X}$ & $\mathrm{X}$ & $\mathrm{X}$ & & \\
\hline & S7 & AQ12 & $\mathrm{X}$ & $\mathrm{X}$ & $\mathrm{X}$ & $\mathrm{X}$ & & \\
\hline & S10 & SL1 & $\mathrm{X}$ & $\mathrm{X}$ & $\mathrm{X}$ & $\mathrm{X}$ & & $\mathrm{X}$ \\
\hline & S15 & RS1 & & $X$ & & $\mathrm{X}$ & & \\
\hline & S14 & AQ19 & $\mathrm{X}$ & $\mathrm{X}$ & $\mathrm{X}$ & $\mathrm{X}$ & & $\mathrm{X}$ \\
\hline & S18 & LS2 & & $\mathrm{X}$ & & $\mathrm{X}$ & $\mathrm{X}$ & $\mathrm{X}$ \\
\hline & No. of samples & 10 & 8 & 9 & 6 & 10 & 1 & 3 \\
\hline \multirow[t]{12}{*}{ Ethene } & S1 & AQ1 & $\mathrm{X}$ & $X$ & $X$ & $\mathrm{X}$ & & $\mathrm{X}$ \\
\hline & S2 & AQ2 & & $\mathrm{X}$ & & $\mathrm{X}$ & & \\
\hline & S3 & AQ5 & $\mathrm{X}$ & & & $\mathrm{X}$ & $\mathrm{X}$ & \\
\hline & S4 & AQ7 & & & & $\mathrm{X}$ & & \\
\hline & S6 & AQ10 & $\mathrm{X}$ & $\mathrm{X}$ & & $\mathrm{X}$ & & \\
\hline & S7 & AQ11 & $\mathrm{X}$ & & $\mathrm{X}$ & $\mathrm{X}$ & & \\
\hline & S9 & AQ13 & $\mathrm{X}$ & $X$ & $X$ & $\mathrm{X}$ & & $\mathrm{X}$ \\
\hline & S8 & GW8 & $\mathrm{X}$ & $\mathrm{X}$ & $\mathrm{X}$ & $\mathrm{X}$ & $\mathrm{X}$ & $\mathrm{X}$ \\
\hline & S11 & SL2 & $\mathrm{X}$ & $\mathrm{X}$ & $\mathrm{X}$ & $\mathrm{X}$ & $\mathrm{X}$ & $\mathrm{X}$ \\
\hline & S12 & SL3 & & $\mathrm{X}$ & & & & \\
\hline & S14 & AQ15 & & $\mathrm{X}$ & $X$ & $\mathrm{X}$ & & $\mathrm{X}$ \\
\hline & No. of samples & 11 & 7 & 8 & 6 & 10 & 3 & 5 \\
\hline
\end{tabular}

${ }^{a}$ End product observed in enrichment culture after several months of incubation

${ }^{\mathrm{b}}$ Information about the sites and samples is given in Online Resource $\times$ Table S1

indicated that either both populations dechlorinated chloroethenes in parallel or that there was a stepwise catalysis where one population dechlorinated the higher chlorinated ethenes (PCE, TCE) and the other the lower chlorinated ones (DCE, VC). Several studies have presented evidence that PCE and TCE dechlorination to ethene has been the result of the activity of different populations successively catalyzing the different dechlorination steps, either by multiple Dehalococcoides spp. populations with different metabolic properties (Duhamel et al. 2002; Waller et al. 2005; Holmes et al. 200; Behrens et al. 2008) or by populations of two or three different genera (Yang et al. 2005; Grostern and Edwards 2006; Amos et al. 2009). In analogy, it is postulated that in the numerous enrichments obtained in this study the dechlorination of PCE was catalyzed first by Desulfitobacterium spp. followed by 

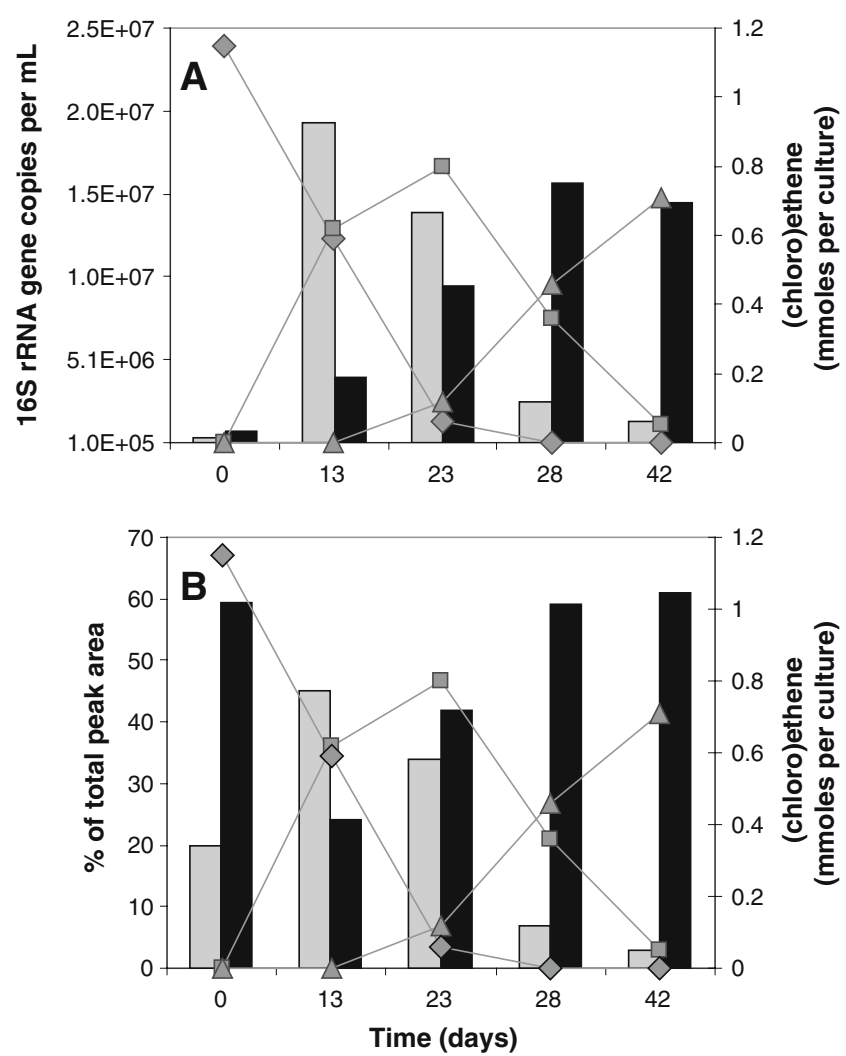

Fig. 2 Number of $16 \mathrm{~S}$ rRNA gene copies per $\mathrm{mL}$ of culture as determined by qPCR (a) and relative population sizes as determined by T-RFLP (b) of Desulfitobacterium spp. and Dehalococcoides spp. in an AQ1 enrichment culture dechlorinating cis-DCE to ethene with propionate-butyrate-ethanol as electron donors. For Dehalococcoides spp. the relative peak area of T-RF 168 is depicted, for Desulfitobacterium spp. the peak area represents the sum of the peaks of T-RF 212 and T-RF 329. Gray bars, Desulfitobacterium spp.; black bars, Dehalococcoides spp.; filled diamond, cis-DCE; filled square, VC; filled triangle, ethene

dechlorination of the lower chlorinated ethenes catalyzed by Dehalococcoides spp. However, dechlorination by Desulfitobacterium spp. was perhaps not only restricted to PCE and TCE.

In all but one enrichment cultures where dechlorination was observed, Desulfitobacterium spp. was detected as very abundant population. This genus was also detected in more than $50 \%$ of the inoculum samples that originated from different European countries (Germany, The Netherlands, and Switzerland). The possible ubiquity of this bacterial genus has already been suggested in a study of soil samples from the Canadian province of Quebec (Lanthier et al. 2001) and presence of Desulfitobacterium spp. in chlorothene-dechlorinating microbial communities has also been reported in several other studies (Davis et al. 2002; Yang et al. 2005; Yoshida et al. 2007; Dowideit et al. 2010).

Besides Desulfitobacterium spp., mainly Dehalococcoides spp. was detected in the enrichment cultures on PCE independently of the dechlorination end product observed.
The first survey on the presence of Dehalococcoides spp. at chloroethene-contaminated sites has shown that this bacterial genus is widely distributed and that it has not been detected in samples from sites at which only incomplete dechlorination occurred (Hendrickson et al. 2002). A link between complete chloroethene dechlorination and the presence of Dehalococcoides spp. has also been presented in numerous other studies investigating enrichment cultures and field sites (Duhamel et al. 2002; Freeborn et al. 2005; Yang et al. 2005; Grostern and Edwards 2006; Holmes et al. 2006; Daprato et al. 2007; Lee et al. 2008; Dowideit et al. 2010). In many enrichment cultures of the present study where Dehalococcoides spp. was detected in the inoculation material, dechlorination was incomplete and stopped at DCE or VC. This could be explained by the fact that in several Dehalococcoides spp.-containing consortia different strains of this bacterial genus seemed to be present with different metabolic properties (Duhamel et al. 2002; Waller et al. 2005; Holmes et al. 2006; Daprato et al. 2007; Lee et al. 2008). Hence, it seems that the mere detection of the presence of Dehalococcoides spp. in a microbial community does not guarantee that dechlorination of chloroethenes will be complete.

In three of the 11 enrichment cultures producing ethene, Dehalococcoides spp. was not detected and also not in two inocula. This suggested that there are other bacteria not affiliated with this genus that are capable to dechlorinate chloroethene to ethene. The identification of a novel group of bacteria within the phylum Chloroflexi as responsible organism for PCE dechlorination (Kittelmann and Friedrich 2008) and of Dehalogenimonas lykanthroporepellens as novel genus and species using polychlorinated alkanes as electron acceptor (Moe et al. 2009) indicates that the phylum Chloroflexi might contain other yet unidentified organohalide-respiring bacteria producing ethene from chloroethenes. Another possible explanation for not detecting Dehalococcoides spp. is, however, that members of this genus were present but not detected by the specific primers used.

The analysis of the AQ1 enrichment culture on cis-1,2DCE suggested based on relative abundances of Desulfitobacterium spp. and Dehalococcoides spp. during dechlorination that cis-DCE was first reduced to VC by both genera, followed by $\mathrm{VC}$ dechlorination by the latter population. This was rather surprising since all described isolates of the genus Desulfitobacterium that dechlorinate PCE and/or TCE produce exclusively cis-DCE (Smidt and de Vos 2004; Villemur et al. 2006). Moreover and regarding the Dehalococcoides spp. population, the VC reductive dehalogenase genes $v c r A$ and $b v c A$ could not be detected in this enrichment (data not shown). Attempts to isolate the different populations present in the AQ1 enrichment on cisDCE did unfortunately fail so far but will be continued. 
Future efforts will in addition concentrate on the identification of the reductive dehalogenase genes involved in complete chloroethene dechlorination by this enrichment.

Acknowledgments This work was supported by the Swiss National Science Foundation grants 3,152-55,413 and 3,100-066,957. We are grateful to Emmanuelle Rohrbach-Brandt and Muriel Gaillard for the technical support and to the collaborators from the EU FP5 project MAROC, especially Janneke Krooneman from Bioclear BV, Groningen, The Netherlands, for supplying many samples used for enrichment inoculation and the collaboration with the primer development and testing.

\section{References}

Altschul SF, Gish W, Miller W, Myers EW, Lipman DJ (1990) Basic Local Alignment Search Tool. J Mol Biol 215(3):403-410

Amos BK, Sung Y, Fletcher KE, Gentry TJ, Wu WM, Criddle CS, Zhou J, Löffler FE (2007) Detection and quantification of Geobacter lovleyi strain SZ: implications for bioremediation at tetrachloroethene- and uranium-impacted sites. Appl Environ Microbiol 73(21):6898-6904

Amos BK, Suchomel EJ, Pennell KD, Löffler FE (2009) Spatial and temporal distributions of Geobacter lovleyi and Dehalococcoides spp. during bioenhanced PCE-NAPL dissolution. Environ Sci Technol 43(6):1977-1985

Behrens S, Azizian MF, McMurdie PJ, Sabalowsky A, Dolan ME, Semprini L, Spormann AM (2008) Monitoring abundance and expression of Dehalococcoides species chloroethene-reductive dehalogenases in a tetrachloroethene-dechlorinating flow column. Appl Environ Microbiol 74(18):5695-5703

Cheng D, Chow WL, He J (2010) A Dehalococcoides-containing coculture that dechlorinates tetrachloroethene to trans-1, 2dichloroethene. ISME J 4(1):88-97

Comber S, Gardner M (1999) An assessment of trends in European environmental data for mercury and chlorinated organic compounds in water and biota. Sci Total Environ 243(244):193-201

Daprato RC, Loffler FE, Hughes JB (2007) Comparative analysis of three tetrachloroethene to ethene halorespiring consortia suggests functional redundancy. Environ Sci Technol 41(7):2261-2269

Davis JW, Odom JM, DeWeerd KA, Stahl DA, Fishbain SS, West RJ, Klecka GM, DeCarolis JG (2002) Natural attenuation of chlorinated solvents at Area 6, Dover Air Force Base: characterization of microbial community structure. J Contam Hydrol 57 (1-2):41-59

Dowideit K, Scholz-Muramatsu H, Miethling-Graff R, Vigelahn L, Freygang M, Dohrmann AB, Tebbe CC (2010) Spatial heterogeneity of dechlorinating bacteria and limiting factors for in situ trichloroethene dechlorination revealed by analyses of sediment cores from a polluted field site. FEMS Microbiol Ecol 71 (3):444-459

Duhamel M, Wehr SD, Yu L, Rizvi H, Seepersad D, Dworatzek S, Cox EE, Edwards EA (2002) Comparison of anaerobic dechlorinating enrichment cultures maintained on tetrachloroethene, trichloroethene, cis-dichloroethene and vinyl chloride. Water Res 36(17):4193-4202

Fetzner S (1998) Bacterial dehalogenation. Appl Microbiol Biotechnol 50(6):633-657

Freeborn RA, West KA, Bhupathiraju VK, Chauhan S, Rahm BG, Richardson RE, Alvarez-Cohen L (2005) Phylogenetic analysis of TCE-dechlorinating consortia enriched on a variety of electron donors. Environ Sci Technol 39(21):8358-8368
Gerritse J, Drzyzga O, Kloetstra G, Keijmel M, Wiersum LP, Hutson R, Collins MD, Gottschal JC (1999) Influence of different electron donors and acceptors on dehalorespiration of tetrachloroethene by Desulfitobacterium frappieri TCE1. Appl Environ Microbiol 65(12):5212-5221

Griffin BM, Tiedje JM, Loffler FE (2004) Anaerobic microbial reductive dechlorination of tetrachloroethene to predominately trans-1, 2-dichloroethene. Environ Sci Technol 38(16):43004303

Grostern A, Edwards EA (2006) Growth of Dehalobacter and Dehalococcoides spp. during degradation of chlorinated ethanes. Appl Environ Microbiol 72(1):428-436

Hendrickson ER, Payne JA, Young RM, Starr MG, Perry MP, Fahnestock S, Ellis DE, Ebersole RC (2002) Molecular analysis of Dehalococcoides 16 S ribosomal DNA from chloroethenecontaminated sites throughout north America and Europe. Appl Environ Microbiol 68(2):485-495

Holliger C, Hahn D, Harmsen H, Ludwig W, Schumacher W, Tindall B, Vazquez F, Weiss N, Zehnder AJB (1998) Dehalobacter restrictus gen. nov. and sp. nov., a strictly anaerobic bacterium that reductively dechlorinates tetra- and trichloroethene in an anaerobic respiration. Arch Microbiol 169(4):313-321

Holliger C, Wohlfarth G, Diekert G (1999) Reductive dechlorination in the energy metabolism of anaerobic bacteria. FEMS Microbiol Rev 22:383-398

Holmes VF, He JZ, Lee PKH, Alvarez-Cohen L (2006) Discrimination of multiple Dehalococcoides strains in a trichloroethene enrichment by quantification of their reductive dehalogenase genes. Appl Environ Microbiol 72(9):5877-5883

Kittelmann S, Friedrich MW (2008) Identification of novel perchloroethene-respiring microorganisms in anoxic river sediment by RNA-based stable isotope probing. Environ Microbiol 10(1):31-46

Lanthier M, Villemur R, Lepine F, Bisaillon JG, Beaudet R (2001) Geographic distribution of Desulfitobacterium frappieri PCP-1 and Desulfitobacterium spp. in soils from the province of Quebec, Canada. FEMS Microbiol Ecol 36(2-3):185-191

Lee PKH, Macbeth TW, Sorenson KS, Deeb RA, Alvarez-Cohen L (2008) Quantifying genes and transcripts to assess the in situ physiology of Dehalococcoides spp. in a trichloroethenecontaminated groundwater site. Appl Environ Microbiol 74 (9):2728-2739

Maidak BL, Cole JR, Parker CT Jr, Garrity GM, Larsen N, Li B, Lilburn TG, McCaughey MJ, Olsen GJ, Overbeek R, Pramanik S, Schmidt TM, Tiedje JM, Woese CR (1999) A new version of the RDP (Ribosomal Database Project). Nucleic Acids Res 27 (1): $171-173$

Maillard J, Schumacher W, Vazquez F, Regeard C, Hagen WR, Holliger C (2003) Characterization of the corrinoid iron-sulfur protein tetrachloroethene reductive dehalogenase of Dehalobacter restrictus. Appl Environ Microbiol 69(8):4628-4638

Maymo-Gatell X, Nijenhuis I, Zinder SH (2001) Reductive dechlorination of cis-1, 2-dichloroethene and vinyl chloride by Dehalococcoides ethenogenes. Environ Sci Technol 35(3):516521

McCarty PL (1997) Breathing with chlorinated solvents. Science 276:1521

Miller GS, Milliken CE, Sowers KR, May HD (2005) Reductive dechlorination of tetrachloroethene to trans-dichloroethene and cis-dichloroethene by PCB-dechlorinating bacterium DF-1. Environ Sci Technol 39(8):2631-2635

Moe WM, Yan J, Nobre MF, da Costa MS, Rainey FA (2009) Dehalogenimonas lykanthroporepellens gen. nov., sp nov., a reductively dehalogenating bacterium isolated from chlorinated solvent-contaminated groundwater. Int J Syst Evol Microbiol 59:2692-2697 
Nijenhuis I, Nikolausz M, Koth A, Felfoldi T, Weiss H, Drangmeister J, Grossmann J, Kästner M, Richnow HH (2007) Assessment of the natural attenuation of chlorinated ethenes in an anaerobic contaminated aquifer in the Bitterfeld/Wolfen area using stable isotope techniques, microcosm studies and molecular biomarkers. Chemosphere 67(2):300-311

Smidt H, de Vos WM (2004) Anaerobic microbial dehalogenation. Annu Rev Microbiol 58:43-73

Smits TH, Devenoges C, Szynalski K, Maillard J, Holliger C (2004) Development of a real-time PCR method for quantification of the three genera Dehalobacter, Dehalococcoides, and Desulfitobacterium in microbial communities. J Microbiol Meth 57(3):369-378

Tamura K, Dudley J, Nei M, Kumar S (2007) MEGA4: Molecular Evolutionary Genetics Analysis (MEGA) software version 4.0. Mol Biol Evol 24(8):1596-1599

Thompson JD, Higgins DG, Gibson TJ (1994) Clustal w: improving the sensitivity of progressive multiple sequence alignment through sequence weighting, positions-specific gap penalties and weight matrix chloice. Nucleic Acids Res 22:4673-4680

Villemur R, Lanthier M, Beaudet R, Lépine F (2006) The Desulfitobacterium genus. FEMS Microbiol Rev 30(5):706-733

Waller AS, Krajmalnik-Brown R, Löffler FE, Edwards EA (2005) Multiple reductive-dehalogenase-homologous genes are simultaneously transcribed during dechlorination by Dehalococcoides-containing cultures. Appl Environ Microbiol 71 (12):8257-8264

Yang YR, Pesaro M, Sigler W, Zeyer J (2005) Identification of microorganisms involved in reductive dehalogenation of chlorinated ethenes in an anaerobic microbial community. Water Res 39(16):3954-3966

Yoshida N, Asahi K, Sakakibara Y, Miyake K, Katayama A (2007) Isolation and quantitative detection of tetrachloroethene (PCE)dechlorinating bacteria in unsaturated subsurface soils contaminated with chloroethenes. J Biosci Bioeng 104(2):91-97 